\title{
A cohort study of the effectiveness of insecticide- treated bed nets to prevent malaria in an area of moderate pyrethroid resistance, Malawi
}

Kim A Lindblade ${ }^{1 *}$, Dyson Mwandama², Themba Mzilahowa ${ }^{2}$, Laura Steinhardt ${ }^{1}$, John Gimnig ${ }^{1}$, Monica Shah ${ }^{1}$, Andy Bauleni' ${ }^{2}$, Jacklyn Wong ${ }^{1}$, Ryan Wiegand ${ }^{1}$, Paul Howell', John Zoya ${ }^{3}$, John Chiphwanya ${ }^{3}$ and Don P Mathanga ${ }^{2}$

\begin{abstract}
Background: Insecticide-treated bed nets (ITNs) are the cornerstone of malaria control in sub-Saharan Africa but their effectiveness may be compromised by the spread of pyrethroid resistance among malaria vectors. The objective of this investigation was to assess the effectiveness of ITNs to prevent malaria in an area of Malawi with moderate pyrethroid resistance.

Methods: One deltamethrin ITN was distributed in the study area for every two individuals in each household plus one extra ITN for households with an odd number of residents. A fixed cohort of 1,199 children aged six to 59 months was seen monthly for one year and at sick visits to measure malaria infection and use of ITNs.

Insecticide resistance among malaria vectors was measured. The effect of ITN use on malaria incidence was assessed, adjusting for potential confounders using generalized estimating equations accounting for repeated measures.

Results: There were 1,909 infections with Plasmodium falciparum over 905 person-years at risk (PYAR), resulting in an observed incidence of 2.1 infections per person-year (iPPY). ITNs were used during 97\% of the PYAR. The main vector was Anopheles funestus: mortality in WHO tube assays after exposure to 0.05\% deltamethrin was 38\% (95\% confidence interval (Cl) 29-47), and resistance was due to elevated oxidase enzymes. After adjusting for potential confounders, the incidence of malaria infection among ITN users was 1.7 iPPY (95\% Cl 1.5-2.1) and among non-bed net users was 2.6 iPPY (95\% Cl 2.0-3.3). Use of ITNs reduced the incidence of malaria infection by $30 \%$ (rate ratio 0.7 ; $95 \% \mathrm{Cl}, 0.5-0.8)$ compared to no bed nets.

Conclusion: ITNs significantly reduced the incidence of malaria infection in children in an area with moderate levels of pyrethroid resistance and considerable malaria transmission. This is the first study to show that ITNs provide protection in areas where pyrethroid-resistant An. funestus is the major malaria vector. Malaria control programmes should continue to distribute and promote ITNs in areas with low to moderate pyrethroid resistance; however, insecticide resistance may intensify further and it is not known whether ITNs will remain effective at higher levels of resistance. There is an urgent need to identify or develop new insecticides and technologies to limit the vulnerability of ITNs to insecticide resistance.
\end{abstract}

Keywords: Malaria, Insecticide-treated bed nets, Prevention, Vector control, Insecticide resistance

\footnotetext{
*Correspondence: kil2@cdc.gov

${ }^{1}$ Division of Parasitic Diseases and Malaria, US Centers for Disease Control and Prevention, 1600 Clifton Rd. NE MS A-06, Atlanta, GA 30333, USA Full list of author information is available at the end of the article
} 


\section{Background}

Bed nets have been used as a physical barrier to prevent nuisance mosquito biting since the Sixth Century BC, but were not used extensively for malaria control until after pyrethroid insecticides were applied to net material in the mid-1980s [1,2]. The combination of the insecticidal and irritant effect of the pyrethroids with the physical barrier of the bed net was found to reduce vector density, sporozoite rates, malaria parasite prevalence, disease incidence, and all-cause child mortality when evaluated both in clinical trials [3] and as part of routine public health programmes [4] in areas where the principal malaria vectors are largely endophagic (biting indoors) and endophilic (resting indoors). As a result, insecticide-treated bed nets (ITNs) are now the cornerstone of malaria prevention in Africa [5]. The World Health Organization (WHO) estimated that between 2010 and 2012, approximately 300 million ITNs were distributed in Africa, at a cost of more than US\$1 billion for their purchase and distribution [6].

Pyrethroids are currently the only class of insecticide recommended for use on ITNs due to their low mammalian toxicity and long residual activity [7]. Concern over the potential impact of pyrethroid resistance on ITN effectiveness was expressed early in the development of ITNs for malaria control [8]. However, after manufacturing processes were developed to mass produce longlasting ITNs, the potential risk from development of insecticide resistance was considered less important than protecting as many people as possible with a 'brilliant' new intervention [5]. As a result, household ITN ownership increased from less than $5 \%$ of sub-Saharan African households in 2000 to almost 60\% in 2012 [6].

Pyrethroid resistance was first reported in Anopheles gambiae s.s. in West Africa in the early 1990s [9], and was later detected in Anopheles funestus and implicated in an epidemic of malaria in South Africa following the switch from DDT to pyrethroids for indoor residual spraying (IRS) [10]. Pyrethroid resistance in both vectors has since spread throughout the continent [11], at least partially as a result of widespread distribution of ITNs [12]. Pyrethroid resistance in anopheline mosquitoes may be due to target site resistance (point mutations that prevent insecticide from binding with receptor molecules on mosquito neurons); metabolic resistance (increased level of one or more enzymes capable of detoxifying or sequestering insecticides); or a combination of the two mechanisms $[11,13]$. Resistant mosquitoes are not killed or knocked down after contact with pyrethroid insecticides. As a result, although ITNs might continue to prevent blood feeding through the insecticide's irritant properties and the bed net's physical barrier, the community effect, which is dependent on the ability of ITNs to kill large numbers of adult mosquitoes, is likely to be compromised.
While there has been some limited evidence linking the operational failure of pyrethroid-based IRS to resistant vectors [14-16], there are as yet no epidemiologic data demonstrating that pyrethroid resistance reduces the effectiveness of ITNs to prevent malaria infection. Several studies of ITN efficacy or effectiveness conducted in pyrethroid-resistant areas have shown continued ability of ITNs to protect against malaria transmission when properly deployed [17-19]. A recent meta-analysis of entomologic outcomes from experimental hut trials conducted in areas with pyrethroid-resistant mosquitoes found that ITNs continued to reduce blood feeding and increase mosquito mortality compared to untreated bed nets, even in the areas with the highest levels of resistance [20]. However, the resistance profiling of the mosquitoes in these studies was inadequate, leaving significant uncertainties as to the resistance mechanisms responsible and thus limiting conclusions.

Malaria remains a significant problem in Malawi, where the entire population is at risk and it was estimated that $48 \%$ of the population resides in areas where the age standardized Plasmodium falciparum prevalence in children aged two to ten years is 40-50\% [21] and more than 6.7 million clinical malaria cases occurred in 2010 [22]. The Malawi National Malaria Control Strategy 2011-2015 allocates almost one-third of its total five-year budget to prevention of malaria transmission through ITNs [23]. Since 2009, more than 20 million ITNs have been distributed in the country through mass campaigns as well as routine mechanisms such as antenatal and well-child clinics. As a result, household ownership of at least one ITN increased from $27 \%$ in 2004 to $55 \%$ in 2012 , and use of ITNs by children less than five years old the night before the survey increased from $<5 \%$ in 2000 to over $55 \%$ in 2012 [22].

WHO phenotypic resistance assays conducted in Malawi in 2007 found both major malaria vectors ( $A n$. funestus and An. gambiae s.l.) to be susceptible to all insecticides, including pyrethroids. Within three years, however, anopheline resistance to pyrethroids could be found in eight of 11 sites examined down the length of the country [24]. The frequency of resistance was higher in An. funestus than An. gambiae s.l.; biochemical assays demonstrated that the resistance mechanism was metabolically based for both species.

National malaria parasite prevalence in children less than five years in Malawi fell in 2012 to 28\% from $43 \%$ in 2010 [25,26]. However, prior to the encouraging 2012 results, several reports suggested that national scale-up of control activities had failed to substantially change the malaria burden in the country [21,27], and pyrethroid resistance was mentioned as a possible threat to successful malaria control [24]. Given the important role for ITNs in the Malawi national malaria control strategy, an 
evaluation was conducted of the effectiveness of ITNs in reducing malaria incidence among a cohort of children six to 59 months old in an area with moderate pyrethroid resistance.

\section{Methods \\ Study area}

The study was conducted in the Traditional Authority of Sitola in Machinga District, surrounding the town of Liwonde (500 $\mathrm{m}$ above sea level; $\left.-15^{\circ} 3^{\prime} \mathrm{S} 35^{\circ} 14^{\prime} \mathrm{E}\right)$. The study area is bordered on the west by the Shire River and on the northeast by the Likwenu River and Liwonde National Park (Figure 1). The population of the area is mostly of the Yao ethnic group, but both Chichewa and Chiyao are spoken. Annual rainfall is unimodal and approximately $1,000 \mathrm{~mm}$ falls between October and May, with daily temperature averaging $26^{\circ} \mathrm{C}$ in the rainy season and $21^{\circ} \mathrm{C}$ in the dry season. In this area, malaria transmission is intense and perennial, peaking during the rainy season. Entomological monitoring in Machinga District by the Malaria Alert Centre of the Malawi College of Medicine (Blantyre, Malawi) prior to the start of the study found a moderate level of pyrethroid resistance of $A n$. funestus to $0.05 \%$ deltamethrin (38\% mortality, 95\% confidence interval (CI) 28-49) (TM, pers comm). This area has never received indoor residual spraying for malaria control.

\section{Study design and population}

The incidence of malaria infection was compared between users and non-users of ITNs in a fixed cohort of children aged six to 59 months who were followed for 12 months after they were cleared of infection with artemether-lumefantrine. Preliminary household mapping was conducted in late 2011 and the six villages closest to Liwonde town, but excluding the urban area itself, were selected for inclusion. Households in these villages were geocoded in February and March 2012 and all children six to 59 months old were invited to participate in the survey, with some households contributing more than one eligible child. Incident cases of malaria infection were identified through a combination of active (routine monthly visits) and passive (sick visits) surveillance.

\section{ITN distribution}

The initial household census was used to determine the number of ITNs needed per house by allocating one ITN per two household residents plus one extra for households with odd numbers of residents. The long-lasting bed nets distributed were Permanet 2.0 (Vestergaard, Lausanne, Switzerland), treated with deltamethrin, and a total of 5,146 ITNs were distributed to household heads during the month of May 2012. The allocation algorithm used in the area was the same as that used by the National Malaria
Control Programme in the national ITN distribution that took place in July 2012 (JZ, pers comm).

\section{Sample size determination}

The sample size was based on a generalized estimating equations modelling approach with a Poisson distribution and accounted for the correlated nature of repeated measurements on study children using an exchangeable correlation structure [28]. The incidence among children who did not use ITNs (projected to be $30 \%$ of the population after the ITN distribution) was estimated to be 1.0 episodes per child-year, and the power to detect a $30 \%$ reduction in incidence among those using ITNs (projected to be $70 \%$ of the population after ITN distribution) was set at $70 \%$, with the probability of committing a Type 1 error equal to $5 \%$. The resulting calculation yielded 684 ITN users and 297 non-ITN users for a total of 981 children. Assuming a 5\% refusal rate, 15\% lossto-follow-up over 12 months, and a mortality rate of $1.5 \%$ per year resulted in a total of 1,335 children to be enrolled at baseline. Under the assumption that in the study area, $60 \%$ of households had at least one child aged six to 59 months, a total of 2,225 households would be needed. As 2,300 households were mapped in the study area, all age-eligible children were invited to participate in the cohort study.

\section{Enrolment and monthly visits}

Children were eligible to participate if they were between six and 59 months as of 1 March, 2012, not taking daily cotrimoxazole for HIV infection or exposure, weighed $5 \mathrm{~kg}$ or more and planned to stay in the study area for at least one month.

At enrolment and all monthly visits, children and their caregivers were invited to a central location near their home; no reimbursement or incentive was provided to caregivers to attend the routine monthly visits. At enrolment and the tenth monthly visit, children had their height and weight measured. At each monthly visit, axillary temperature was measured and a finger stick was performed to collect a blood sample for thick and thin blood smears and haemoglobin $(\mathrm{Hb})$ measurement using

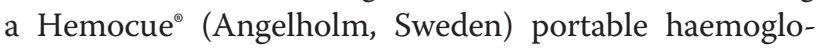
binometer. At enrolment, a dried blood spot was also made for Plasmodium testing by nested polymerase chain reaction (PCR) at the Malawi College of Medicine in Blantyre [29]. All samples collected at enrolment were tested for the presence of P. falciparum, Plasmodium malariae, Plasmodium ovale, and Plasmodium vivax. At monthly visits, malaria testing was conducted using a histidine-rich protein 2 (HRP-2) P. falciparum malaria rapid diagnostic test (RDT; SD Bioline Malaria Ag Pf $^{\bullet}$ ref. 05FK53, Kyonggi-do, Republic of Korea). 


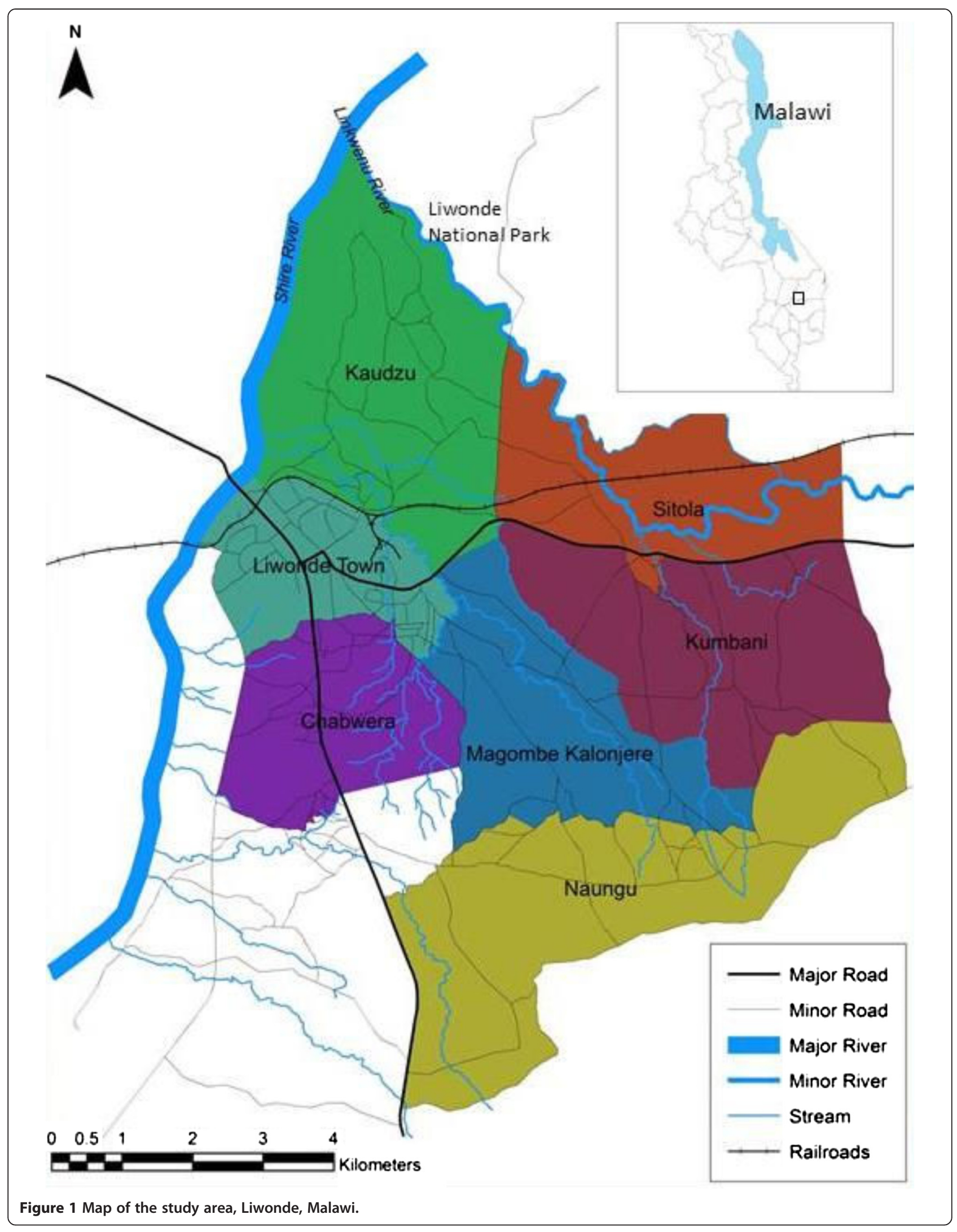


At enrolment and all monthly visits, parents or caregivers were questioned regarding the child's two-week illness history, household bed net ownership and bed net use. Bed net use by the child was referenced for the night before the survey, the two-week period before the survey and for typical use during the current season. Study ITNs were not distributed until after enrolment in May 2012, and many residents retained their older bed nets. Therefore, the age of the bed net used by the child was requested along with the caregiver's assessment of the condition (presence/absence of holes, the number of holes larger than a fist and the number larger than a head).

At enrolment, all children were provided with a full, weightappropriate treatment course of dispersible artemetherlumefantrine (Coartem ${ }^{\circledR}$-D, Novartis, Basel, Switzerland); the first dose was observed by study staff, and parents or caregivers were instructed on how and when to give the remaining doses. At subsequent visits, only children with positive RDT results were treated with an appropriate anti-malarial.

Study staff attempted to contact any child who did not attend the monthly visit to schedule a make-up visit. Any child who did not attend three consecutive visits for any reason was withdrawn from the study and censored as of their last visit date.

\section{Sick visits}

During the study period, caregivers were encouraged to bring participants to the study clinic located at Machinga District Hospital, the only health care facility within $30 \mathrm{~km}$ of Liwonde Town, if they became sick. Artemisinin-based combination therapy is free at public health facilities, and $91 \%$ of children under five years of age with fever in the two weeks before the Malawi Malaria Indicator Survey in 2012 and treated with an antimalarial received an artemisinin-based combination therapy, suggesting limited use of private physicians and drug shops [26]. Sick children were examined by a study clinician who measured axillary temperature and took a finger-prick blood sample for malaria testing using a RDT, and $\mathrm{Hb}$ testing with a Hemocue ${ }^{\bullet}$ The same questionnaire used at enrolment and the monthly visits was employed to elicit a two-week period illness history and bed net use from the child's caregiver. Caregivers were reimbursed for transport to the study clinic for one sick visit per month.

\section{Confirmation of ITN use}

The reliability of caregiver-reported ITN use was evaluated twice during the study period (JW, pers comm). Briefly, children attending monthly visits between December 2012 and January 2013 and September and October 2013 were randomly selected for follow-up home visits, which occurred between zero and eight days after the monthly visit.
Caregiver responses to questions about ITN use at the monthly visits were compared to the same questions asked at the home visit plus visual inspection of the ITN reported to be used by the child. The proportion of positive and negative agreement of ITN use between caregiver report and home visit were calculated and 95\% CI were constructed using bootstrapping.

\section{Entomologic surveillance and resistance monitoring}

The study area was divided into ten clusters and every two weeks pyrethroid spray catches (PSCs) were conducted in ten houses per cluster using standard methods [30]. Sexing and morphologic species identification was conducted in the laboratory at the Malaria Alert Centre. Anopheles funestus and An. gambiae s.l. mosquitoes were tested for the presence of sporozoites in their salivary glands using standard procedures [31]. Phenotypic pyrethroid resistance was evaluated in the study area during the course of the study using the standard WHO tube assay with two to five days old An. funestus and An. gambiae s.l. reared from eggs or larvae [32].

To determine whether the mechanism of resistance was an elevated oxidase or esterase enzyme, mosquitoes were pre-exposed to the synergist, piperonyl butoxide (PBO), for one hour before the WHO tube assay was conducted. Synergists are non-toxic to insects but improve the effect of an insecticide by inhibiting the metabolic enzymes (oxidases and esterases) that cause mosquitoes to be resistant. Mosquitoes were exposed to PBO in $250 \mathrm{ml}$ Wheaton bottles. The bottles were coated by adding $1 \mathrm{ml}$ of a stock solution of $400 \mathrm{mg} / \mathrm{L}$ of $\mathrm{PBO}$ (corresponding to a dose of $400 \mathrm{ml} /$ bottle) in acetone, rotating the bottles to ensure all surfaces were covered and then allowing the acetone to evaporate. Two to five days old female mosquitoes were added to the bottle and exposed for one hour. The mosquitoes were then removed and exposed to $0.05 \%$ deltamethrin or $0.75 \%$ permethrin in WHO tube assays as described above [33].

Assays were performed to detect mutations in the knockdown resistance $(k d r)$ locus using either a leg or wing removed from the mosquito corpse of mosquitoes for which species identification was confirmed molecularly. Stratified samples of mosquitoes of both species determined by the WHO resistance assay to be resistant or susceptible to permethrin or deltamethrin were randomly selected for testing for commonly reported $k d r$ mutations. Two different assays were utilized depending on the species being tested: Huynh et al. for An. gambiae s.l. and Morgan et al. for An. funestus [34,35]. All assays were performed without modification and PCR amplicons were visualized on a $1.5 \% 0.5 \mathrm{X}$ tris-borate-EDTA agarose gel stained with ethidium bromide and visualized in a Gel Doc- $\mathrm{It}^{2}$ UV imager (UVP LLC, Upland, CA, USA). Further sequencing was done on An. funestus samples to 
determine if single nucleotide polymorphisms associated with resistant populations were present within the amplified region. Samples were processed using BigDye ${ }^{\circ}$ Terminator v3.1 (Life Technologies, Grand Island, NY, USA), purified with BigDye ${ }^{\bullet}$ XTerminator and sequenced on an ABI 3500 sequencer (Applied Biosystems, Foster City, CA, USA). Sequences were aligned using Lasergene ${ }^{\circ}$ SeqMan (DNASTAR, Madison, WI, USA) and confirmed using the basic local alignment search tool feature.

The WHO definition considers insecticide resistance to be confirmed when mortality of mosquitoes exposed to insecticides in bioassays is $<90 \%$ [32]. The level of resistance was classified according to Strode et al. [20].

Mosquitoes of both species (An. funestus and An. gambiae s.l.) were selected from the mosquitoes alive or killed by the resistance bioassay and tested using PCR to determine the sibling species. Mosquitoes of the An. gambiae complex were identified using the methods described by Wilkins et al. [36] while members of the An. funestus group were identified using a modification of the protocol described by Koekemoer et al. with intentional mismatched primers [37,38]. For An. funestus, specimens were tested with primers for An. funestus s.s., Anopheles vaneedeni, Anopheles rivulorum, An. rivulorum-like, Anopheles parensis, and Anopheles leesoni. Anopheles gambiae s.l. were tested with primers for An. gambiae s.s., Anopheles arabiensis, Anopheles coluzzii, Anopheles quadriannulatus (species A) and Anopheles melas/merus. Mosquitoes that did not amplify against one of these primers were retested with universal primers for the internal transcribed spacer 2 region to determine whether the lack of amplification was due to poor specimen preservation.

\section{Data management and analysis}

The main outcome was $P$. falciparum parasitaemia at a sick or monthly visit as measured by RDT. The main exposure variable was bed net use the night before the visit, categorized as ITN, untreated bed net (UTN) or no bed net. Bed nets used between enrolment and the end of the ITN distribution were classified as a UTN if they were reported to be 36 months or older; however, as of 1 June, 2012, after ITNs were distributed by the project, no bed nets were considered to be untreated. Children were described as anaemic $(\mathrm{Hb}<11 \mathrm{~g} / \mathrm{dl})$ or not anaemic $(\mathrm{Hb} \geq 11 \mathrm{~g} / \mathrm{dl})$.

A number of potential confounders was measured and included in the analysis. Baseline parasitaemia was measured by PCR and used to indicate previous exposure to malaria infection. A proxy measure of individual exposure to malaria transmission was generated by using the inverse distance-weighted (IDW) malaria prevalence in children within $1 \mathrm{~km}$, divided into terciles [39]. Household altitude was divided into terciles. Household ownership of key assets was analysed using principal component analysis and the coefficient of the first component was used to generate a household asset score [40]. Assets included electricity, paraffin lamp, battery lamp radio, television, cell phone, mattress, sofa set, table and chairs, refrigerator, bicycle, motorcycle, car, source of water, type of toilet, type of floor material, type of roof material, type of wall material and number of sleeping rooms. Children were divided into terciles according to their household's asset score. Stunting (height-for-age) and wasting (weight-for-age) were defined as Z-scores $<-2$ compared to the WHO growth reference charts by age and sex [41]. Children were remeasured in the tenth month of the survey and their nutritional status was allowed to change. Because of the potential for the density of ITNs around a participant to create a mass effect, participants were divided into terciles based on the total number of ITNs found in households within $300 \mathrm{~m}$; this radius was based on data from a cluster-randomized trial of ITNs in western Kenya that found a mass effect within $300 \mathrm{~m}$ [42]. Data on household ITN ownership from the household mapping exercise before the start of the study were used to calculate ITN density for participants between enrolment and the time of the ITN distribution. After the ITN distribution was completed, a new density calculation was made assuming the number of ITNs per household matched the distribution algorithm of one ITN per every two household residents with one extra for households with an odd number of residents. The seasonality of malaria transmission was accounted for by dummy variables indicating visits during the high (AprilJune 2012 and January-March 2013) or low (July-December 2012) malaria transmission season.

Data from monthly and sick visits were combined into one dataset and time between visits was calculated as person-time at risk. After every positive result treated with artemether-lumefantrine, including the baseline treatment dose, 10.5 days were subtracted from the person-time at risk to account for the half-life of lumefantrine [43].

As febrile children who came for sick visits in between monthly visits would have been tested again for malaria with an RDT, there was the potential that the second of sequentially positive RDTs was a false positive due to persistent antigen from a recently treated infection. Followup of infected children after malaria treatment in Uganda found that the median duration of persistent antigenaemia using HRP2-based RDTs was 35 days (95\% CI 33-37), although the possibility of re-infection could not be ruled out [44]. A mathematical model of HRP2 antigenaemia found that persisting antigen caused tests to remain positive for up to seven days after treatment, depending on the length and density of infection before treatment [45]. In this study, there were 972 pairs of sequential visits (out of a total of 13,166 visits) in children with positive RDT results. The median time between these paired, positive sequential visits was 17 days. To attempt to minimize the 
potential that the second of these two visits was a false positive, second visits $>=17$ days were considered new infections, whereas second visits with a positive RDT that occurred $<17$ days after an earlier positive visit were considered false positives. Recognizing that there is no clear cut-off to determine whether a second positive RDT was due to a new infection or persistent antigenaemia, a sensitivity analysis was conducted by varying the cut-off $+/$-one week in either direction, i.e., cut-offs of 10 and 24 days.

$\mathrm{SAS}^{\bullet}$ (v. 9.3, SAS Institute, Inc., Cary, NC, USA) was used for all analyses. Data were analysed using Poisson regression with a generalized estimating equations approach (PROC GENMOD) to account for the extra correlation from repeated measures on the same child [46]. Both the exposure variable and covariates were allowed to be time varying. Log-transformed person-time was used as an offset and an exchangeable working correlation structure was specified. Any covariate with a p-value of $>0.1$ in univariate analysis was included in the multivariate model. Rate ratios (RR) and 95\% CI were calculated from model parameters and model-adjusted incidence rates for covariates are presented. Protective effectiveness (PE) was calculated as $100 \% *\left(1-R_{1} / R_{0}\right)$ where $R_{1}$ is the rate among ITN or UTN users and $R_{0}$ is the rate among non-bed net users. The attributable rate difference was calculated as $\mathrm{R}_{1}-\mathrm{R}_{0}$ and interpreted as the number of malaria infections prevented by ITNs annually.

\section{Human subjects}

Household heads provided verbal consent to have their household mapped and residents enumerated. Parents or caregivers provided verbal consent to have their child screened for eligibility, and read and signed written informed consent forms if the child was found to be eligible. Households selected for PSCs provided verbal consent. The protocol was reviewed and approved by the Malawi College of Medicine Research Ethics Committee (Blantyre, Malawi) and by the US Centers for Disease Control and Prevention institutional review board (Atlanta, GA, USA).

\section{Results}

\section{Study profile and baseline characteristics}

There were 2,804 houses in the study area, and 2,178 (78\%) agreed to be mapped and enumerated (Figure 2). A total of 1,665 children between six and 59 months old were registered and 1,199 (72\%) enrolled from 907 households. There were 268 (29\%) households with more than one child enrolled. A total of 175 (10\%) children could not be located during the enrolment period or were found to have moved out of the study area; 201 (12\%) children declined to participate, and 90 (5\%) were found to be ineligible. Comparing the enrolled children with those who declined to participate or could not be located, males

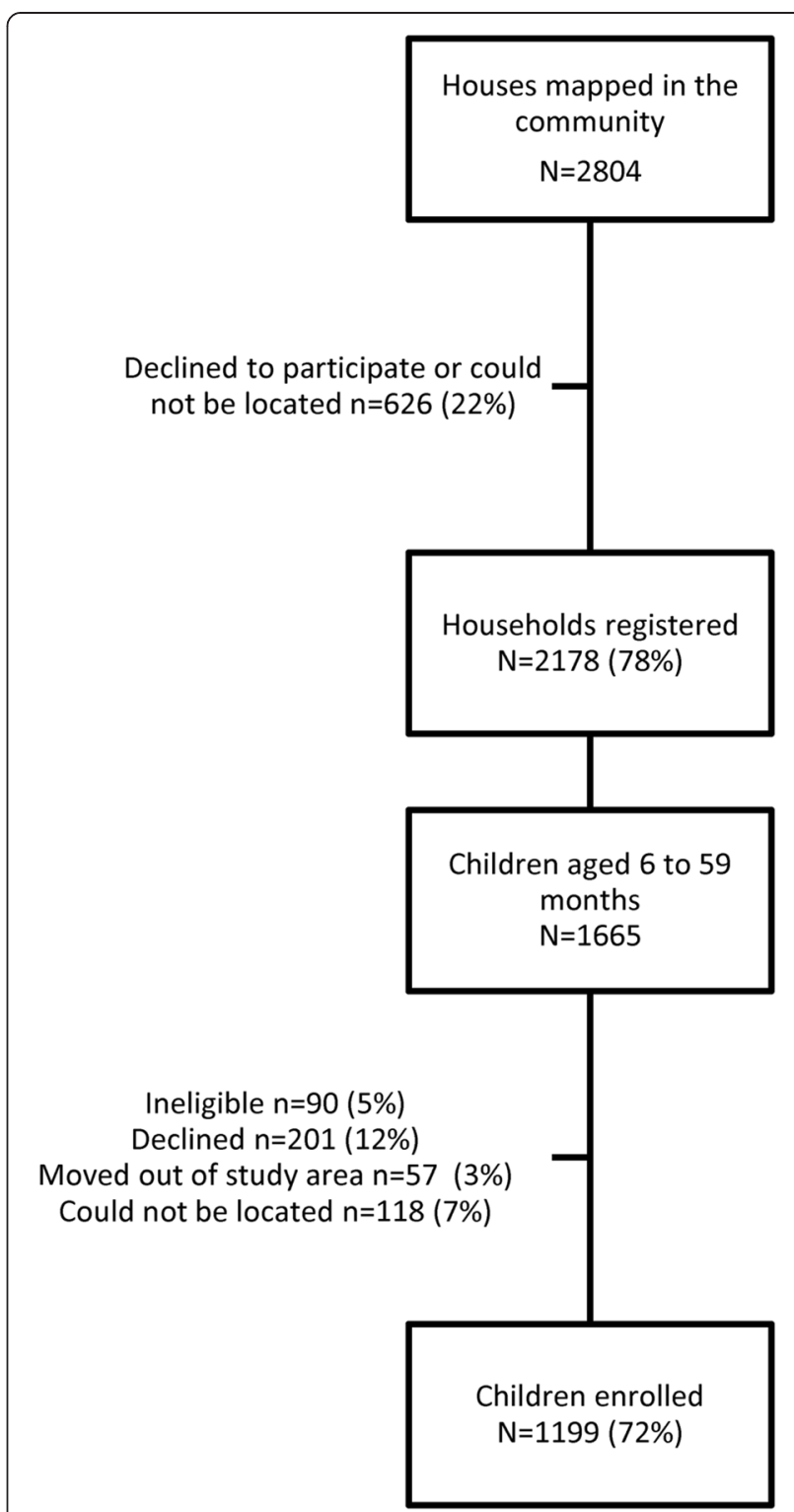

Figure 2 Study profile, Liwonde, Malawi 2012-2013.

(78\%) were more likely to be enrolled than females (74\%; Chi square test $\mathrm{p}=0.04)$, but those enrolled had a similar median age (30 months) to those not enrolled (29 months; Wilcoxon signed-rank test $\mathrm{p}=0.80$ ). There was no significant difference in enrolment between children who reported sleeping under a bed net $(76 \%)$ and those who did not $(73 \%$; Chi square test $\mathrm{p}=0.26)$.

Median follow-up time of the cohort of 1,199 children was 0.96 years (interquartile range (IQR) $0.92,0.97$ ) and 1,018 (85\%) children completed one year of follow-up (Table 1). Reasons for early exit from the cohort included: consent withdrawn or child moved out of the study area $(\mathrm{n}=145,12 \%)$, started cotrimoxazole therapy $(\mathrm{n}=3,0.2 \%)$, died $(\mathrm{n}=2,0.2 \%)$, and could not be traced 


\begin{tabular}{|c|c|}
\hline Characteristic & Results \\
\hline Number of children enrolled & 1,199 \\
\hline Number of households included & 907 \\
\hline Median age at enrolment in months (IQR)* & $30(17,44)$ \\
\hline $\begin{array}{l}\text { Number of children who completed one } \\
\text { year follow-up, } n(\%)\end{array}$ & $1,018(85)$ \\
\hline Person-years at risk & 905 \\
\hline Female, n (\%) & $579(48)$ \\
\hline Plasmodium infection at baseline (PCR), $\mathrm{n} / \mathrm{N}(\%)^{*}$ & $440 / 1,192(37)$ \\
\hline Median IDW malaria prevalence $<1 \mathrm{~km}(\mathrm{IQR})^{*}$ & $35 \%(24,48)$ \\
\hline Anaemic ( $\mathrm{Hb}<11 \mathrm{~g} / \mathrm{dl})$, n (\%) & $860(72)$ \\
\hline Caregiver completed primary school, n (\%) & $215(18)$ \\
\hline Bed net used, n/N (\%) & $922 / 1,175(78)$ \\
\hline ITN used, n/N (\%) & $516 / 1,174(44)$ \\
\hline ITN has $\geq 1$ hole fist-sized or larger, n/N (\%) & $110 / 404(21)$ \\
\hline \multicolumn{2}{|l|}{ Median number of ITNs within 300 m (IQR) } \\
\hline Baseline & $8(5,21)$ \\
\hline After ITN distribution & $48(27,116)$ \\
\hline Malaria infections** & 1,909 \\
\hline Median malaria incidence per person-year (IQR) & $1.2(0,3.6)$ \\
\hline
\end{tabular}

*IQR = Interquartile range; $\mathrm{PCR}=$ polymerase chain reaction; IDW = inverse distance-weighted; $\mathrm{Hb}=$ haemoglobin; $\mathrm{PPY}=$ per person year.

${ }^{* *}$ As measured by malaria rapid diagnostic tests.

( $\mathrm{n}=31,3 \%$ ). The participants had 13,166 encounters with the study and the time at risk for the whole cohort was 905 person-years (after removing non-risk periods due to anti-malarial treatment). Attendance at monthly visits varied between 90\% in May 2012 and 74\% in December 2012; at least one sick visit was made by $67 \%(n=803)$ of the participants, and the median number of sick visits among them was 2 (IQR 1, 4).

Slightly less than half (48\%) of the enrolled children were female (Table 1). At baseline, 37\% of children were infected with malaria as confirmed by PCR, the majority (97\%) with $P$. falciparum, and the remainder with $P$. malariae and $P$. ovale. The median IDW prevalence of malaria in children within $1 \mathrm{~km}$ was $35 \%$ (IQR 24, 48). The median $\mathrm{Hb}$ measurement was $10.0 \mathrm{~g} / \mathrm{dl}$ (IQR $8.7,11.1)$ and $72 \%$ of children were anaemic. A third of children (32\%) were stunted and 3\% met the definition of wasted. Less than one-fifth (18\%) of the caregivers had completed primary school.

At baseline, bed net use the night before the survey was reported for $78 \%$ of children, but only $44 \%$ were reported to have used an ITN. Use of an ITN the night before the survey was strongly associated with use of an ITN over the previous fortnight: $97 \%$ of those who used an ITN the night before the survey had used an ITN every night out of the previous 14 nights and the remainder had used an ITN at least once in the last two weeks. Among those reporting not using an ITN the night before the survey, 99\% reported not using an ITN at all during the previous 14 nights. A large proportion (59\%) of the ITNs were reported to have holes, with one-fifth $(21 \%)$ reported to have at least one hole fistsized or greater. The median number of ITNs within $300 \mathrm{~m}$ of participants at baseline was 8 (IQR 5, 21).

\section{Malaria incidence}

Between April 2012 and March 2013 there were 1,909 infections with $P$. falciparum identified, most (65\%) through active surveillance at monthly visits (Table 1 ). The median incidence per child was 1.2 infections per person-year (iPPY) (IQR 0, 3.6), and the overall incidence was 2.1 iPPY. A third of the children (32\%) never had documented malaria infection during the study, whereas $44 \%$ had two or more infections. During the study period, which crossed two rainy seasons, there were two peaks of transmission corresponding to the end of the 2012 rainy season (May 2012) and the last month of the 2013 rainy season included in the study survey (March 2013; Figure 3).

\section{Use of ITNs over time}

Information on child's ITN use was recorded for 895 person-years at risk (PYAR), and ITN use the night before the survey was reported for 864 (97\%) PYAR, UTN use for 17 (2\%) PYAR and no bed net for 15 (2\%) PYAR. To measure the reliability of caregiver-reported ITN use at the routine monthly surveys, study staff visited 211 randomly selected children from December 2012January 2013 and 325 children between September and October 2013 (JW, pers comm). The observed proportions of positive agreement between caregiver report and home visit on ITN use by the child in the first and second surveys were $98.8 \%$ (95\% CI 97.6-99.8) and 93.3\% (95\% CI 91.2-95.3), respectively. The proportions of negative agreement in the first and second survey were 28.6\% (95\% CI 0-75) and 20.0\% (95\% CI 0.1-35.0).

\section{Entomologic surveillance and resistance monitoring}

There were 885 female anophelines collected through PSC; 743 (84\%) were identified as An. funestus Giles and 142 (16\%) as An. gambiae s.l. Mean indoor resting density for both species was highest in April 2012 and again between December 2012 and March 2013 (Figure 4). A total of 711 of the An. funestus and 114 of the An. gambiae s.l. collected by PSCs were tested for sporozoites and 30 (4\%) and two (2\%) were positive, respectively.

Results of the phenotypic resistance assays are shown in Table 2. Mortality of An. funestus after exposure to $0.05 \%$ deltamethrin and $0.75 \%$ permethrin was 38 and 


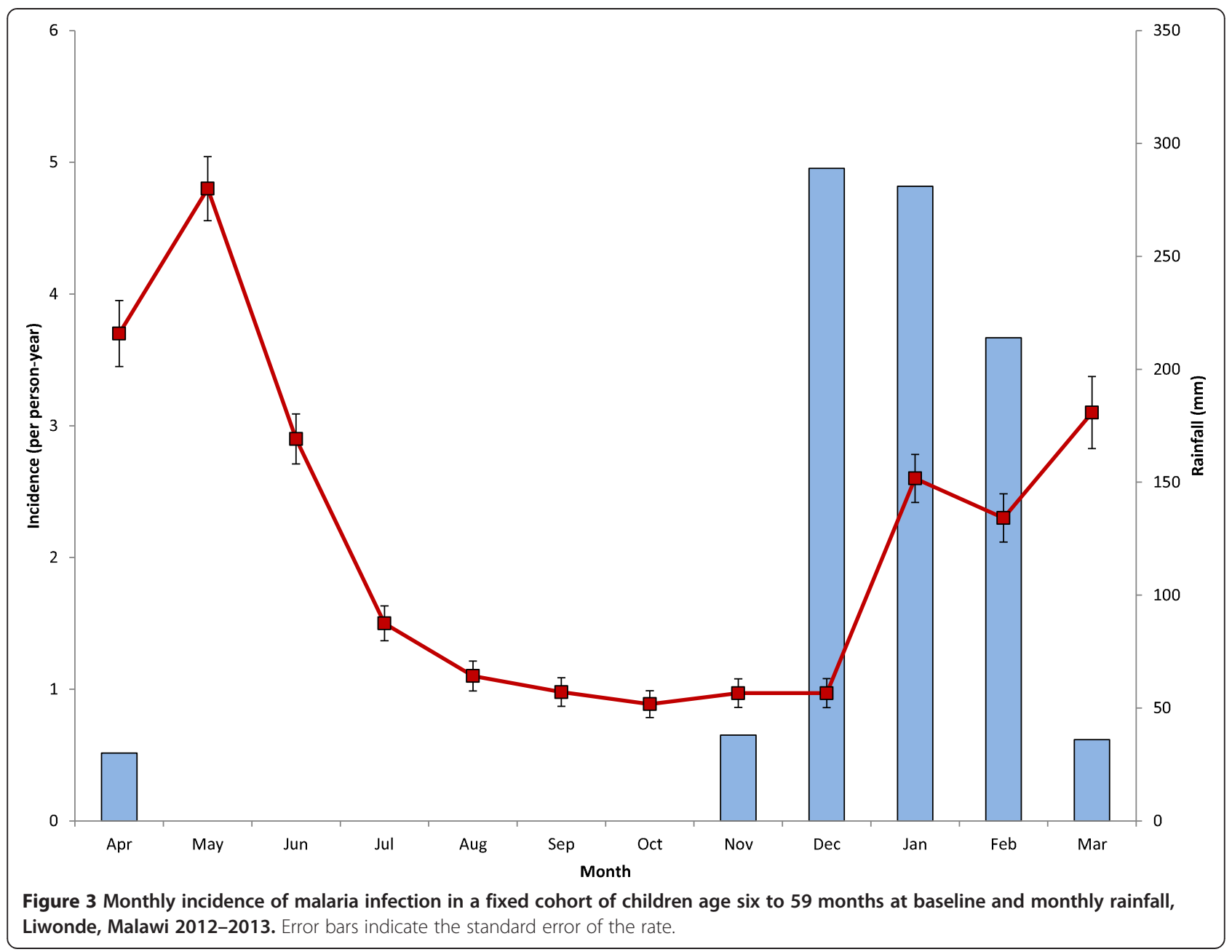

$25 \%$, respectively. After exposure to the synergist (PBO), mortality rates with these insecticides rose to 89 and $98 \%$, respectively. Mortality of An. gambiae s.l. to $0.05 \%$ deltamethrin and $0.75 \%$ permethrin was 53 and $65 \%$, and exposure to $\mathrm{PBO}$ increased mortality from $0.75 \%$ permethrin to $100 \%$. No testing of An. gambiae s.l. to $0.05 \%$ deltamethrin after PBO exposure was conducted due to low numbers of mosquitoes. Taken together, the results of increased pyrethroid susceptibility after exposure to PBO implicate elevated oxidase enzymes as the primary resistance mechanism.

Of the $22 A n$. gambiae s.l. tested, none possessed either of the two $k d r$-like mutations L1014F (West African $k d r$ ) or L1014S (East African $k d r$ ) reported in An. gambiae s.s. Of the $20 \mathrm{An}$. funestus samples chosen for additional sequencing, seven resistant and eight susceptible samples yielded complete sequences. Based on alignments with the $A n$. funestus FUMOZ laboratory colony, which originates from Mozambique and does not possess $k d r$, none had any single nucleotide polymorphisms that were unique to either the resistant or susceptible populations.
In summary, neither vector species showed evidence for the presence of $k d r$ genes.

There were 23 An. funestus s.l. identified to species out of 100 that survived exposure to pyrethroids, and 12 tested out of 39 that were killed by exposure to pyrethroid and all (100\%) were found to be An. funestus s.s. There were 17 An. gambiae s.l. tested that survived the pyrethroid exposure and 22 that did not, and 16 and 22, respectively, were identified to species; $100 \%$ of the surviving mosquitoes and 95\% (21/22) of the killed mosquitoes were found to be $A n$. arabiensis. One (5\%) of the killed mosquitoes was identified as An. gambiae s.s.

\section{Bed nets and malaria incidence}

In univariate analysis, children between six and 11 months old experienced twice the malaria incidence than those older than 12 months; malaria infection at baseline was also associated with almost twice the malaria incidence of those without infection at baseline (Table 3). Use of ITNs, but not UTNs, was associated with a $60 \%$ reduction in the incidence of malaria compared to children who did not 


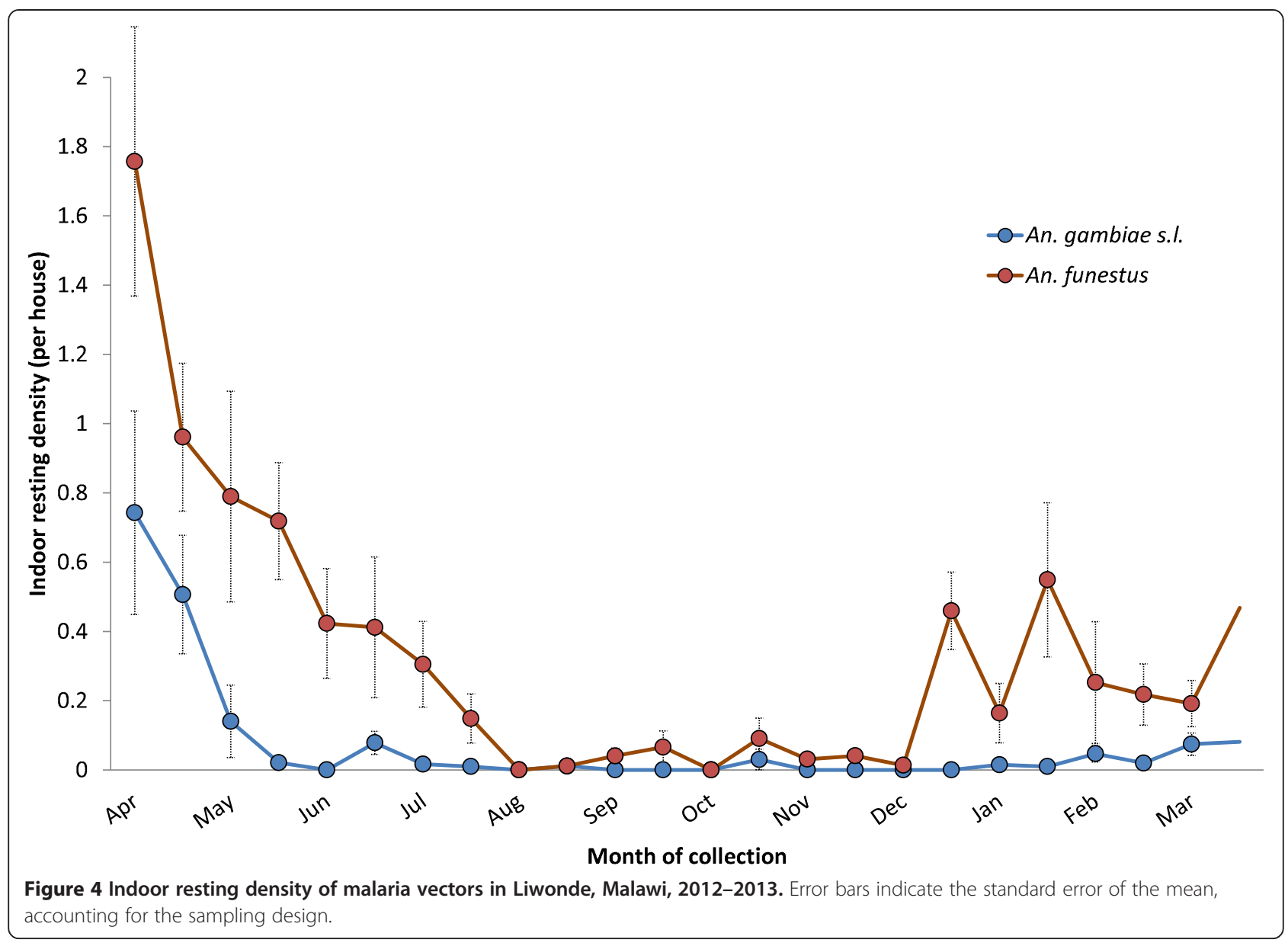

use bed nets. Poorer children and those whose caregivers had not completed primary school had higher rates of malaria. Children living at the lowest altitudes had a 40$60 \%$ increase in malaria incidence compared to those at the highest elevations. Children living in households with the lowest numbers of ITNs within $300 \mathrm{~m}$ experienced almost twice the incidence of malaria than children living with the highest ITN density, whereas the children living with the lowest IDW malaria prevalence within $1 \mathrm{~km}$ experienced $20-40 \%$ reductions in malaria incidence. Malaria incidence was highest after the rains from April to June 2012, with an incidence of $4.1 \mathrm{iPPY}$, and lowest during the dry season from July to December 2012, with an incidence of $0.9 \mathrm{iPPY}$.

After controlling for all variables significantly associated with malaria incidence in univariate analyses, use of an ITN was associated with a protective effectiveness of $30 \%$ (RR 0.7, 95\% CI 0.5-0.8; Table 3). UTNs were not statistically associated with malaria incidence (RR 0.8, 95\% CI 0.6-1.0). Results were not sensitive to the time period used

Table 2 Results of resistance testing using the WHO tube assay with two to five days old mosquitoes with and without pre-exposure to a synergist, Liwonde, Malawi 2012-2013

\begin{tabular}{|c|c|c|c|}
\hline & & Without pre-exposure to $\mathrm{PBO}^{\mathrm{a}}$ & With pre-exposure to $\mathrm{PBO}$ \\
\hline \multirow{2}{*}{$\begin{array}{l}\text { Mosquito } \\
\text { species }\end{array}$} & Insecticide & $\%$ mortality $(95 \% \mathrm{Cl})^{\mathbf{b}}$ & $\%$ mortality $(95 \% \mathrm{Cl})$ \\
\hline & & (No. died/tested) & (No. died/tested) \\
\hline \multirow[t]{2}{*}{ An. funestus } & Deltamethrin $0.05 \%$ & $38(29-47)(58 / 152)$ & $89(37-100)(34 / 38)$ \\
\hline & Permethrin $0.75 \%$ & $25(8-42)(23 / 93)$ & $98(93-100)(89 / 91)$ \\
\hline \multirow[t]{2}{*}{ An. gambiae s.l. } & Deltamethrin $0.05 \%$ & $53(24-81)(49 / 93)$ & \\
\hline & Permethrin $0.75 \%$ & $57(32-82)(65 / 114)$ & $100(90-100)(27 / 27)$ \\
\hline
\end{tabular}

${ }^{\mathrm{a}}$ Mosquitoes with and without pre-exposure to PBO for one hour before resistance testing using the WHO tube assay.

b $95 \%$ confidence intervals calculated taking clustering by tube into account. 
Table 3 Predictors of malaria incidence in a fixed cohort of 1,199 children age six to 59 months at baseline, Liwonde, Malawi 2012- 2013

\begin{tabular}{|c|c|c|c|c|c|c|c|c|c|}
\hline \multirow{2}{*}{ Characteristic } & & \multirow[b]{2}{*}{$\begin{array}{l}\text { Malaria } \\
\text { infections (n) }\end{array}$} & \multirow[b]{2}{*}{$\begin{array}{l}\text { Person-years } \\
\text { at risk }\end{array}$} & \multicolumn{3}{|l|}{ Univariate } & \multicolumn{3}{|l|}{ Multivariate } \\
\hline & & & & $\begin{array}{l}\text { Observed } \\
\text { incidence } \\
\text { PPY }\end{array}$ & $\begin{array}{l}\text { Rate ratio } \\
(95 \% \mathrm{Cl})\end{array}$ & $P$ value & $\begin{array}{l}\text { Adjusted } \\
\text { incidence } \\
\text { PPY }(95 \% \mathrm{Cl})\end{array}$ & $\begin{array}{l}\text { Rate ratio } \\
(95 \% \mathrm{Cl})\end{array}$ & $P$ value \\
\hline \multirow[t]{2}{*}{ Age (months) } & $6-11$ & 64 & 17 & $3.7(2.7-5.0)$ & $2.2(1.6-3.0)$ & $<0.0001$ & $2.1(1.5-2.9)$ & $1.0(0.8-1.4)$ & 0.84 \\
\hline & $12+$ & 1845 & 891 & $1.7(1.6-1.8)$ & 1.0 & & $2.0(1.8-2.3)$ & 1.0 & \\
\hline \multirow[t]{2}{*}{ Sex } & Female & 905 & 433 & $1.7(1.6-1.9)$ & $1.0(0.9-1.1)$ & 0.96 & & & \\
\hline & Male & 1004 & 471 & $1.7(1.6-1.9)$ & 1.0 & & & & \\
\hline \multirow{2}{*}{$\begin{array}{l}\text { Baseline Plasmodium } \\
\text { infection }\end{array}$} & Positive & 948 & 329 & $2.4(2.2-2.7)$ & $1.7(1.5-2.0)$ & $<0.0001$ & $2.7(2.2-3.3)$ & $1.7(1.5-1.9)$ & $<0.0001$ \\
\hline & Negative & 960 & 573 & $1.4(1.3-1.5)$ & 1.0 & & $1.6(1.3-2.0)$ & 1.0 & \\
\hline \multirow[t]{3}{*}{ Bed net use } & ITN & 1710 & 864 & $1.6(1.5-1.7)$ & $0.4(0.3-0.5)$ & $<0.0001$ & $1.7(1.5-2.1)$ & $0.7(0.5-0.8)$ & $<0.0001$ \\
\hline & UTN & 92 & 17 & $4.9(4.1-5.9)$ & $1.1(0.8-1.5)$ & 0.53 & $2.0(1.6-2.6)$ & $0.8(0.6-1.0)$ & 0.06 \\
\hline & No bed net & 86 & 15 & $4.4(3.5-5.7)$ & 1.0 & & $2.6(2.0-3.3)$ & 1.0 & \\
\hline \multirow[t]{3}{*}{ Wealth index } & Poorest & 725 & 290 & $2.1(1.9-2.3)$ & $1.5(1.3-1.8)$ & $<0.0001$ & $2.3(1.9-2.8)$ & $1.3(1.1-1.5)$ & 0.0008 \\
\hline & Middle & 647 & 296 & $1.8(1.6-2.0)$ & $1.3(1.1-1.6)$ & 0.001 & $2.2(1.8-2.7)$ & $1.2(1.1-1.5)$ & 0.01 \\
\hline & Least poor & 495 & 309 & $1.4(1.2-1.6)$ & 1.0 & & $1.8(1.4-2.2)$ & 1.0 & \\
\hline \multirow{2}{*}{$\begin{array}{l}\text { Caregiver completed } \\
\text { primary }\end{array}$} & Yes & 239 & 164 & $1.2(0.9-1.4)$ & $0.6(0.5-0.8)$ & $<0.0001$ & $1.9(1.5-2.4)$ & $0.8(0.7-1.0)$ & 0.02 \\
\hline & No & 1628 & 730 & $1.9(1.7-2.0)$ & 1.0 & & $2.3(1.9-2.8)$ & 1.0 & \\
\hline \multirow[t]{3}{*}{ Altitude } & Lowest & 680 & 300 & $1.9(1.7-2.1)$ & $1.4(1.2-1.7)$ & 0.0001 & $2.4(1.9-2.9)$ & $1.5(1.3-1.8)$ & $<0.0001$ \\
\hline & Middle & 727 & 294 & $2.1(1.9-2.4)$ & $1.6(1.3-1.9)$ & $<0.0001$ & $2.4(2.0-2.9)$ & $1.5(1.3-1.8)$ & $<0.0001$ \\
\hline & Highest & 497 & 307 & $1.3(1.2-1.5)$ & 1.0 & & 1. $(1.3-2.0)$ & 1.0 & \\
\hline \multirow[t]{3}{*}{ Number of ITNs $<300 \mathrm{~m}$} & Lowest & 586 & 249 & $2.0(1.8-2.3)$ & $1.7(1.4-2.1)$ & $<0.0001$ & $2.1(1.7-2.6)$ & $1.1(0.9-1.4)$ & 0.39 \\
\hline & Middle & 737 & 313 & $2.1(1.9-2.3)$ & $1.7(1.5-2.1)$ & $<0.0001$ & $2.2(1.8-2.7)$ & $1.2(0.9-1.4)$ & 0.17 \\
\hline & Highest & 389 & 272 & $1.2(1.0-1.4)$ & 1.0 & & $1.9(1.5-2.4)$ & 1.0 & \\
\hline \multirow[t]{3}{*}{ IDW malaria prevalence } & Lowest & 474 & 294 & $1.3(1.1-1.5)$ & $0.6(0.5-0.7)$ & $<0.0001$ & $1.7(1.3-2.1)$ & $0.7(0.5-0.8)$ & 0.0002 \\
\hline & Middle & 610 & 304 & $1.7(1.5-1.9)$ & $0.8(0.6-0.9)$ & 0.0003 & $2.2(1.7-2.7)$ & $0.9(0.7-1.0)$ & 0.04 \\
\hline & Highest & 820 & 302 & $2.3(2.1-2.5)$ & 1.0 & & $2.5(2.1-3.1)$ & 1.0 & \\
\hline \multirow[t]{3}{*}{ Season } & Apr-June & 844 & 178 & $4.1(3.8-4.4)$ & $2.3(2.0-2.7)$ & $<0.0001$ & $4.6(3.9-5.5)$ & $2.3(2.0-2.6)$ & $<0.0001$ \\
\hline & July-Dec & 556 & 486 & $0.9(0.8-1.0)$ & $0.5(0.4-0.6)$ & $<0.0001$ & $1.0(0.8-1.2)$ & $0.5(0.4-0.6)$ & $<0.0001$ \\
\hline & Jan-Mar & 509 & 241 & $1.8(1.6-2.0)$ & 1.0 & & $2.0(1.6-2.5)$ & 1.0 & \\
\hline
\end{tabular}

${ }^{a}$ PPY = per person year; IDW = inverse distance-weighted; ITN = insecticide-treated bed net; UTN = untreated bed net; $\mathrm{Cl}=$ confidence interval.

${ }^{b}$ Incidence is model-adjusted for all other variables in the column.

to define whether the second of two sequential visits with positive RDT results was considered a new infection: with a cut-off of 10 days, use of an ITN was associated with a $30 \%$ reduction in malaria incidence ( $R R$ 0.7, 95\% CI 0.5 0.9 ), whereas a cut-off of 24 days resulted in a $40 \%$ reduction (RR 0.6, 95\% CI 0.5-0.7).

The model-adjusted incidence of malaria among ITN users was 1.7 iPPY (95\% CI 1.5-2.1) and among UTN users was 2.0 iPPY (95\% CI 1.6-2.6), whereas among children who did not use any bed net, the model-adjusted incidence was $2.6 \mathrm{iPPY}$ (95\% CI 2.0-3.3). The attributable rate difference for ITNs (subtracting the incidence rate among those who used ITNs from the incidence rate of those who did not, i.e. 2.6-1.7 iPPY) was 0.9 malaria infections averted per person-year, or 815 malaria infections averted in the study population. Therefore, for every ten children protected by ITNs, nine malaria infections were prevented annually.

\section{Discussion}

ITNs were found to reduce the incidence of malaria by $30 \%$ among children six to 59 months old in an area of Malawi where deltamethrin killed only $38 \%$ of $A n$. funestus, the main malaria vector, and 53\% of An. gambiae s.l., the secondary vector, in WHO tube assays. Prior to the continent-wide increase in pyrethroid resistance, randomized controlled trials of the efficacy of ITNs compared to no bed nets in areas with similar malaria incidence to Malawi showed $\geq 50 \%$ reductions in the incidence of malaria [47-49]. It is possible, therefore, that in the absence of pyrethroid resistance, ITN effectiveness might have been higher in this population. However, the lower 
effectiveness of ITNs in this study compared to earlier trials also could be due to factors related to the study design (e.g., observational cohort $v s$ randomized controlled trial) and analytical methods. Additionally, the high rate of ITN use in this population may have suppressed transmission, benefitting non-bed net users and reducing differences between users and non-users of ITNs.

ITNs prevent malaria through several modes of action: the insecticide deters mosquitoes from entering houses, irritates the mosquitoes that encounter the insecticide, causing them to leave the area prematurely, and kills the mosquitoes that acquire a lethal dose. In addition, ITNs provide a physical barrier to blood feeding. This latter mode of action is the only one that does not depend on the insecticide, and potentially could account for continued ITN effectiveness in areas with pyrethroid resistance if the barrier effect was an important component of the ITN mode of action. However, evidence of the effectiveness of UTNs to reduce malaria transmission is limited: several observational studies have found UTNs to be protective against malaria [50], but the only communityrandomized controlled trial of UTNs compared to no bed nets did not find an epidemiologic benefit, although there was a reduction in blood feeding $[51,52]$. In this study, there was no significant difference in the incidence of malaria between children using UTNs compared to children not using bed nets, but because UTNs were present in the study community prior to the start of the study, the integrity of their barrier may have been reduced through acquisition of holes.

There are other data to suggest that ITNs provide more than just a physical barrier to malaria transmission in areas with resistant mosquitoes. A recent meta-analysis of the entomologic impacts of pyrethroid resistance found that ITNs were significantly more effective at reducing blood feeding, inducing exophily and killing mosquitoes than UTNs despite pyrethroid resistance [20]. This review found that significant heterogeneity in methods and results across the studies evaluated made it impossible to determine whether ITN effectiveness was affected by the level of insecticide resistance, but in conjunction with the results of this study, it appears reasonable to conclude that the insecticide on ITNs continues to exert a beneficial effect even when a significant degree of pyrethroid resistance is present [20]. Whether the beneficial effect could have been larger in the absence of pyrethroid resistance is not clear.

This is the first study to show that ITNs continue to prevent malaria infections in an area where pyrethroid-resistant An. funestus is the dominant vector. As the clearest example to date of operational failure of insecticide-based vector control was in South Africa after An. funestus became metabolically resistant to the pyrethroids used in the IRS programme in the late 1990s [10], the results from this study hint at potential differences in the impact of insecticide resistance on ITNs and IRS. As vector control interventions, ITNs and IRS both deter mosquitoes from entering houses, induce exophily and kill mosquitoes that come into contact with the insecticide. However, ITNs also act as baited traps, luring mosquitoes to the insecticide with vapour trails of human body gasses [53], and there are differences between ITNs and IRS in terms of the concentration of insecticide used and the duration of the insecticide that might affect results. It is conceivable that these differences could mitigate the impact of insecticide resistance on ITNs but not IRS. Alternatively, it is possible that the failure of IRS in South Africa was not only due to insecticide resistance: increases in malaria parasite resistance to the first-line anti-malarial treatment (sulphadoxine-pyrimethamine) occurred around the same time and may have played a role in the resurgence of cases [54]. Unfortunately, the available data are not sufficient to clarify the situation.

A weakness in all studies of insecticide resistance and malaria transmission is the actual measurement of resistance. WHO recommends measurement of phenotypic resistance through standardized WHO tube assays or the CDC bottle assay [32]. Both tests measure mosquito mortality after a period of exposure to insecticide. It is recommended to use recently emerged, non-blood fed, adult female mosquitoes to standardize comparisons over time and between sites. However, results from these laboratory assays may not be generalizable to older mosquitoes with infective sporozoites. As they age, mosquitoes may lose the oxidase or esterase enzymes responsible for detoxifying insecticides and become more susceptible to insecticides than younger mosquitoes [55]. As a result, phenotypic assays of recently emerged mosquitoes may overestimate the degree of resistance in the epidemiologic important vectors. However, a more recent study suggests that blood meals cause oxidative stress in resistant mosquitoes that may maintain resistance within a mosquito throughout its life [56]. Due to the low vector density in the area, it was not possible to compare phenotypic resistance assays in wild-caught and recently emerged mosquitoes contemporaneously to determine whether results differed, but this is an area for future exploration.

Aside from the issue of whether existing assays accurately measure the resistance of the mosquitoes responsible for transmitting malaria, it is not clear whether there is a threshold at which resistance may begin to have an epidemiologic impact. Strode and colleagues classified resistance levels into low, medium and high based on phenotypic assays and prevalence of $k d r$; although this somewhat arbitrary classification system was used in this analysis, it is not based on any epidemiologic or entomologic outcomes [20]. It is conceivable that the level of resistance measured in this study is not severe enough to show important epidemiologic effects. 
Given that resistance levels could increase even more, there will continue to be a need to monitor the impact of resistance on epidemiologic outcomes until and unless, new insecticides or technologies reduce the dependence of ITNs on pyrethroids.

This study had several important limitations. Resistance among mosquito vectors had already occurred in this area so no comparison with ITNs under conditions of full vector susceptibility in this population could be made. ITN use in this population was significantly higher than anticipated, decreasing the precision of the protective effectiveness estimate, and potentially limiting the generalizability of the study results to other areas. The ITNs used in this study were new at the start, and it is possible that effectiveness will decline as bed nets degrade physically. To examine this possibility, the study was extended by an additional nine months; results are being analysed and will be published soon. Use of RDTs for malaria diagnosis during the monthly and sick visits, rather than the gold standard of microscopy, was necessary given the extremely high number of encounters with study participants where malaria infection was measured; but RDTs may pick up treated infections that still have persistent antigen. This problem was assessed by varying the interval for determining whether consecutive positive RDT results were independent, and did not find any difference in interpretation of results.

This study had several strengths, including that it was designed prospectively to answer the question of ITN effectiveness in an area with significant pyrethroid resistance, rather than conceived as a secondary data analysis. A fixed cohort with a high follow-up rate was observed, and ITN use was evaluated frequently over the course of the study with two surveys to examine the reliability of caregiver report of ITN use. Both active and passive surveillance were combined to try to capture all malaria infections, both symptomatic and asymptomatic, and all infections were parasitologically confirmed. Potential confounders were carefully measured and included in the final model, including estimates of malaria exposure and measures of ITN density that may cause a community effect. A recent review lamented the lack of concomitant measures of insecticide resistance in other studies of ITNs and pyrethroid resistance [20]; this study collected information before and during the study to ensure that insecticide resistance measurements were characteristic of the vector species transmitting malaria in the same time and place as the study participants, with the caveat that only recently emerged mosquitoes were evaluated. Additionally, the mechanism of insecticide resistance underpinning the phenotypic results was identified.

\section{Conclusion}

ITNs continue to protect children from malaria in an area of Malawi with very high reported ITN use, moderate insecticide resistance and high rates of malaria transmission, annually preventing nine cases of malaria for every ten children protected. Based on these findings, national malaria control programmes should continue to procure and distribute ITNs for areas with moderate insecticide resistance. However, as the measures of insecticide resistance used in this investigation are imperfect, and distribution and intensity of insecticide resistance could still increase, these findings do not suggest complacency with the status quo. Periodic monitoring of the effectiveness of ITNs in areas of moderate to high pyrethroid resistance should continue, while research on new insecticides and new technologies should be pursued urgently to eliminate the pyrethroid Achilles' heel of ITNs.

\section{Abbreviations}

Cl: Confidence interval; Hb: Haemoglobin; HRP-2: Histidine-rich protein 2; IDW: Inverse-distance weighted; iPPY: Incidence per person-year;

IQR: Interquartile range; ITN: Insecticide-treated bed net; kdr: Knockdown resistance; RDT: Rapid diagnostic test; PBO: Piperonyl butoxide;

PCR: Polymerase chain reaction; PE: Protective effectiveness; PSC: Pyrethroid spray capture; PYAR: Person-years at risk, RR, rate ratio; UTN: Untreated bed net; WHO: World Health Organization.

\section{Competing interests}

The authors declare they have no competing interests.

\section{Authors' contributions}

KAL, JG and DPM conceived of the study. KAL, DM, TM, LS, JG, MS, AB, JW, RW, JZ, JC, and DPM participated in its design and coordination. TM and PH conducted molecular assays on mosquitoes. KAL, LS, MS, PH, and JG helped to draft the manuscript. All authors read and approved the final manuscript.

\section{Acknowledgements}

The authors appreciate the support and cooperation of the Machinga District Hospital management team, traditional chiefs, nurses, interviewers, and all the children and caregivers who participated in the study. The authors thank Adam Wolkon for his excellent support to field data collection activities in mapping, enrolment and monthly interviews. Gerard W Lopez prepared the map. The study was made possible with funding from the US President's Malaria Initiative and CDC through Cooperative Agreement No. 3 U01CK000135 between the CDC and the University of Malawi, College of Medicine. The findings and conclusions in this report are those of the authors and do not necessarily represent the views of the Centers for Disease Control and Prevention.

\section{Author details}

${ }^{1}$ Division of Parasitic Diseases and Malaria, US Centers for Disease Control and Prevention, 1600 Clifton Rd. NE MS A-06, Atlanta, GA 30333, USA.

${ }^{2}$ Malaria Alert Centre, Malawi College of Medicine, Blantyre, Malawi. ${ }^{3}$ National Malaria Control Programme, Ministry of Public Health, Lilongwe, Malawi.

Received: 18 October 2014 Accepted: 7 January 2015

Published online: 28 January 2015

\section{References}

1. Travis BV, Morton FA. Treatment of clothing for protection against mosquitoes. Proc Annu Meet N J Mosqu Exterm Assoc. 1946;33:65-9.

2. Graves PM, Brabin BJ, Charlwood JD, Burkot TR, Cattani JA, Ginny M, et al. Reduction in incidence and prevalence of Plasmodium falciparum in under-5-year-old children by permethrin impregnation of mosquito nets. Bull World Health Organ. 1987;65:869-77.

3. Lengeler $C$. Insecticide-treated bednets and curtains for preventing malaria. Cochrane Database Syst Rev 2004;2: CD000363.

4. Lim SS, Fullman N, Stokes A, Ravishankar N, Masiye F, Murray CJ, et al. Net benefits: a multicountry analysis of observational data examining 
associations between insecticide-treated mosquito nets and health outcomes. PLoS Med. 2011;8:e1001091.

5. Eisele TP, Steketee RW. African malaria control programs deliver ITNs and achieve what the clinical trials predicted. PLoS Med. 2011;8:e1001088.

6. World Health Organization. World Malaria Report 2013. Geneva, Switzerland: World Health Organization; 2013.

7. Hougard JM, Duchon S, Darriet F, Zaim M, Rogier C, Guillet P. Comparative performances, under laboratory conditions, of seven pyrethroid insecticides used for impregnation of mosquito nets. Bull World Health Organ. 2003;81:324-33.

8. Quelennec G. Pyrethroids in the WHO pesticide evaluation scheme (WHOPES). Parasitol Today. 1988:4:S15-17.

9. Elissa N, Mouchet J, Riviere F, Meunier JY, Yao K. Resistance of Anopheles gambiae s.s. to pyrethroids in Cote d'Ivoire. Ann Soc Belg Med Trop. 1993;73:291-4.

10. Hargreaves K, Koekemoer LL, Brooke BD, Hunt RH, Mthembu J, Coetzee M. Anopheles funestus resistant to pyrethroid insecticides in South Africa. Med Vet Entomol. 2000;14:181-9.

11. Ranson H, N'Guessan R, Lines J, Moiroux N, Nkuni Z, Corbel V. Pyrethroid resistance in African anopheline mosquitoes: what are the implications for malaria control? Trends Parasitol. 2011;27:91-8.

12. Ndiath MO, Sougoufara S, Gaye A, Mazenot C, Konate L, Faye O, et al. Resistance to DDT and pyrethroids and increased kdr mutation frequency in An. gambiae after the implementation of permethrin-treated nets in Senegal. PLoS One. 2012;7:e31943.

13. Corbel V, N'Guessan R. Distribution, mechanisms, impact and management of insecticide resistance in malaria vectors: a pragmatic review. In: Manguin S, editor. Anopheles mosquitoes - New insights into malaria vectors. 2013. p. 579-633.

14. Maharaj R, Mthembu DJ, Sharp BL. Impact of DDT re-introduction on malaria transmission in KwaZulu-Natal. S Afr Med J. 2005;95:871-4.

15. Casimiro SL, Hemingway J, Sharp BL, Coleman M. Monitoring the operational impact of insecticide usage for malaria control on Anopheles funestus from Mozambique. Malar J. 2007;6:142.

16. Sharp BL, Ridl FC, Govender D, Kuklinski J, Kleinschmidt I. Malaria vector control by indoor residual insecticide spraying on the tropical island of Bioko. Equatorial Guinea Malar J. 2007;6:52

17. Henry MC, Assi SB, Rogier C, Dossou-Yovo J, Chandre F, Guillet P, et al. Protective efficacy of lambda-cyhalothrin treated nets in Anopheles gambiae pyrethroid resistance areas of Cote d'Ivoire. Am J Trop Med Hyg. 2005;73:859-64.

18. Damien GB, Djenontin A, Rogier C, Corbel V, Bangana SB, Chandre F, et al. Malaria infection and disease in an area with pyrethroid-resistant vectors in southern Benin. Malar J. 2010;9:380.

19. Tokponnon FT, Ogouyemi AH, Sissinto Y, Sovi A, Gnanguenon V, Cornelie S, et al. Impact of long-lasting, insecticidal nets on anaemia and prevalence of Plasmodium falciparum among children under five years in areas with highly resistant malaria vectors. Malar J. 2014;13:76.

20. Strode C, Donegan S, Garner P, Enayati AA, Hemingway J. The impact of pyrethroid resistance on the efficacy of insecticide-treated bed nets against African anopheline mosquitoes: systematic review and meta-analysis. PLoS Med. 2014;11:e1001619.

21. Bennett A, Kazembe L, Mathanga DP, Kinyoki D, Ali D, Snow RW, et al. Mapping malaria transmission intensity in Malawi, 2000-2010. Am J Trop Med Hyg. 2013;89:840-9.

22. World Health Organization. Focus on Malawi. In Progress \& Impact Series. Geneva, Switzerland; 2013.

23. National Malaria Control Programme M. Malaria Strategic Plan 2011-2015: Towards Universal Access. Lilongwe, Malawi: Ministry of Health; 2011

24. Wondji CS, Coleman M, Kleinschmidt I, Mzilahowa T, Irving H, Ndula M, et al. Impact of pyrethroid resistance on operational malaria control in Malawi. Proc Natl Acad Sci U S A. 2012;109:19063-70.

25. Ministry of Health. National Malaria Indicator Survey 2010. Lilongwe, Malawi: Malawi Ministry of Health; 2011.

26. Ministry of Health. National Malaria Indicator Survey 2012. Lilongwe, Malawi: Malawi Ministry of Health; 2013.

27. Roca-Feltrer A, Kwizombe CJ, Sanjoaquin MA, Sesay SS, Faragher B, Harrison J, et al. Lack of decline in childhood malaria, Malawi, 2001-2010. Emerg Infect Dis. 2012;18:272-8.

28. Rochon J. Application of GEE procedures for sample size calculations in repeated measures experiments. Stat Med. 1998;17:1643-58.
29. Singh B, Bobogare A, Cox-Singh J, Snounou G, Abdullah MS, Rahman HA. A genus- and species-specific nested polymerase chain reaction malaria detection assay for epidemiologic studies. Am J Trop Med Hyg. 1999;60:687-92.

30. Service MW. Mosquito Ecology: Field Sampling Methods. New York: John Wiley \& Sons; 1976

31. Wirtz RA, Zavala F, Charoenvit Y, Campbell GH, Burkot TR, Schneider I, et al. Comparative testing of monoclonal antibodies against Plasmodium falciparum sporozoites for ELISA development. Bull World Health Organ. 1987;65:39-45.

32. World Health Organization. Test procedures for insecticide resistance monitoring in malaria vector mosquitoes. Geneva, Switzerland: World Health Organization; 2013.

33. Brogdon WG, Chan A. Guideline for evaluating insecticide resistance in vectors using the CDC bottle bioassay; 2010. http://www.cdc.gov/malaria/ resources/pdf/fsp/ir_manual/ir_cdc_bioassay_en.pdf

34. Morgan JC, Irving H, Okedi LM, Steven A, Wondji CS. Pyrethroid resistance in an Anopheles funestus population from Uganda. PLoS One. 2010:5:e11872.

35. Huynh LY, Sandve SR, Hannan LM, Van Ert M, Gimnig JE. Fitness costs of pyrethroid insecticide resistance in Anopheles gambiae. In Annual Meeting of the Society for the Study of Evolution; Christchurch, New Zealand; 2007

36. Wilkins EE, Howell PI, Benedict MQ. IMP PCR primers detect single nucleotide polymorphisms for Anopheles gambiae species identification, Mopti and Savanna rDNA types, and resistance to dieldrin in Anopheles arabiensis. Malar J. 2006;5:125.

37. Koekemoer L. Anopheles funestus complex. In: Benedict MQ, editor. Methods in Anopheles Research. Atlanta, GA: US Centers for Disease Control; 2011.

38. Wilkins EE. Benedict MQ. In: Methods in Anopheles Research. GA: US Centers for Disease Control and Prevention; 2011.

39. Olotu A, Fegan G, Wambua J, Nyangweso G, Ogada E, Drakeley C, et al. Estimating individual exposure to malaria using local prevalence of malaria infection in the field. PLoS One. 2012;7:e32929.

40. Filmer D, Pritchett LH. Estimating wealth effects without expenditure data - or tears: an application to educational enrollments in states of India. Demography. 2001;38:115-32.

41. WHO Multicentre Growth Reference Study Group. WHO Child Growth Standards: Length/height-for-age, weight-for-age, weight-for-length, weight-for-height and body mass index-for-age: Methods and development Geneva, Switzerland: World Health Organization; 2006;312.

42. Hawley WA, Phillips-Howard PA, ter Kuile FO, Terlouw DJ, Vulule JM, Ombok $M$, et al. Community-wide effects of permethrin-treated bed nets on child mortality and malaria morbidity in western Kenya. Am J Trop Med Hyg. 2003;68 Suppl 4:121-7

43. Ezzet F, van Vugt M, Nosten F, Looareesuwan S, White NJ. Pharmacokinetics and pharmacodynamics of lumefantrine (benflumetol) in acute falciparum malaria. Antimicrob Agents Chemother. 2000:44:697-704

44. Kyabayinze DJ, Tibenderana JK, Odong GW, Rwakimari JB, Counihan H. Operational accuracy and comparative persistent antigenicity of HRP2 rapid diagnostic tests for Plasmodium falciparum malaria in a hyperendemic region of Uganda. Malar J. 2008;7:221.

45. Marquart L, Butterworth A, McCarthy JS, Gatton ML. Modelling the dynamics of Plasmodium falciparum histidine-rich protein 2 in human malaria to better understand malaria rapid diagnostic test performance. Malar J. 2012;11:74.

46. Zeger SL, Liang KY. Longitudinal data analysis for discrete and continuous outcomes. Biometrics. 1986:42:121-30

47. ter Kuile FO, Terlouw DJ, Kariuki SK, Phillips-Howard PA, Mirel LB, Hawley WA, et al. Impact of permethrin-treated bednets on malaria, anemia and growth in infants in an area of intense perennial malaria transmission in western Kenya. Am J Trop Med Hyg. 2003;68 Suppl 4:68-77.

48. Marbiah NT, Petersen E, David K, Magbity E, Lines J, Bradley DJ. A controlled trial of lambda-cyhalothrin-impregnated bed nets and/or dapsone/pyrimethamine for malaria control in Sierra Leone. Am J Trop Med Hyg. 1998;58:1-6.

49. Gimnig JE, Vulule JM, Lo TQ, Kamau L, Kolczak MS, Phillips-Howard PA, et al. Impact of permethrin-treated bed nets on entomologic indices in an area of intense year-round malaria transmission. Am J Trop Med Hyg. 2003, 68

50. Guyatt H, Snow RW. The cost of not treating bednets. Trends Parasitol. 2002:18:12-16.

51. Snow RW, Rowan KM, Lindsay SW, Greenwood BM. A trial of bed nets (mosquito nets) as a malaria control strategy in a rural area of The Gambia, West Africa. Trans R Soc Trop Med Hyg. 1988;82:212-5. 
52. Lindsay SW, Shenton FC, Snow RW, Greenwood BM. Responses of Anopheles gambiae complex mosquitoes to the use of untreated bednets in The Gambia. Med Vet Entomol. 1989;3:253-62.

53. Curtis CF, Jana-Kara B, Maxwell CA. Insecticide treated nets: impact on vector populations and relevance of initial intensity of transmission and pyrethroid resistance. J Vector Borne Dis. 2003:40:1-8.

54. Hemingway $\mathrm{J}$. The role of vector control in stopping the transmission of malaria: threats and opportunities. Philos Trans R Soc Lond B Biol Sci. 2014;369:20130431.

55. Rajatileka S, Burhani J, Ranson H. Mosquito age and susceptibility to insecticides. Trans R Soc Trop Med Hyg. 2011;105:247-53.

56. Oliver SV, Brooke BD. The effect of multiple blood-feeding on the longevity and insecticide resistant phenotype in the major malaria vector Anopheles arabiensis (Diptera: Culicidae). Parasit Vectors. 2014;7:390.

\section{Submit your next manuscript to BioMed Central and take full advantage of:}

- Convenient online submission

- Thorough peer review

- No space constraints or color figure charges

- Immediate publication on acceptance

- Inclusion in PubMed, CAS, Scopus and Google Scholar

- Research which is freely available for redistribution 